\title{
UNITARY BORDISM OF CIRCLE ACTIONS
}

\author{
by CZES KOSNIOWSKI and MAHGOUB YAHIA
}

(Received 12th November 1981)

\section{Introduction}

The purpose of this paper is to describe $\mathfrak{A}_{*}^{T}$, the bordism module of unitary $T$ manifolds, where $T$ denotes the circle group $S^{1}$. We give both an algebraic and a geometric description. The algebraic result is

$$
\mathfrak{U}_{*}^{T} \cong \bigoplus \mathfrak{U}_{*}\left(B U_{i(1)} \times B U_{i(2)} \times \cdots \times B U_{i(2 n)}\right)
$$

where $I=(i(1), i(2), \ldots, i(2 n))$ runs through all finite ordered $2 n$-tuples $(n \geqq 0)$ of nonnegative integers which satisfy the conditions $(a) i(1)+i(2 n) \neq 0$ and $(b)$ if $i(2 n) \neq 0$ then $i(2 n) \neq 1$. The isomorphism is also described geometrically and this leads to geometric generators of $\mathfrak{H}_{*}^{T}$.

A. Hattori and $\mathrm{H}$. Taniguchi studied $\mathfrak{U}_{*}^{T}$ in [1], our work sheds new light on their results as well as simplifying some of their descriptions.

\section{Background bordism}

By a manifold we mean a compact (usually closed) smooth unitary manifold and by a $T$-manifold we mean a manifold with a smooth unitary action of $T$. For a more detailed account of what follows in this section (but for unoriented manifolds) we refer the reader to [3].

If $H$ is a subgroup of $T$ then an $H$ module $U$ is a finite dimensional complex vector space together with complex linear action of $H$ on it. If $M$ is a $T$-manifold and $x \in M$ then $T_{x}$, the isotropy subgroup at $x$, is the subgroup of $T$ that fixes $x$ (i.e. $T_{x}=\{t \in T ; t x=x\}$ ). For each $x \in M$ there is a $T_{x}$ module $\bar{V}_{x}$ which is equivariantly diffeomorphic to a $T_{x}$ neighbourhood of $x$. This module $V_{x}^{x}$ decomposes as $\bar{V}_{x}=V_{x} \oplus V_{x}^{\prime}$ where $T_{x}$ acts trivially on $V_{x}^{\prime}$ and no non-zero vector in $V_{x}$ is fixed by all of $T_{x}$ (i.e. $V_{x}$ has no trivial $T_{x}$ submodules). We refer to the pair $\left[T_{x} ; V_{x}\right]$ as the slice-type of $x \in M$. By a $T$-slice type we mean a pair $[H ; U]$ where $H$ is a subgroup of $T$ and $U$ is an $H$ module with no trivial $H$ submodules. A family of $T$-slice types $\mathscr{F}$ is a collection of $T$-slice types satisfying the condition that if $[H ; U] \in \mathscr{F}$ and $x \in T \times{ }_{H} U$ then the slice type $\left[T_{x} ; V_{x}\right]$ at $x$ belongs to $\mathscr{F}$. We say that the $T$-manifold $M$ is of type $\mathscr{F}$ if for all $x \in M$ the slice type $\left[T_{x} ; V_{x}\right] \in \mathscr{F}$. A $T$-manifold-with-boundary $M$ is of type $(\mathscr{F}, \mathscr{F})$ if for all $x \in M$ the slice type $\left[T_{x} ; V_{x}\right] \in \mathscr{F}$. Two $n$-dimensional $T$-manifolds $M_{1}, M_{2}$ of type $\mathscr{F}$ are said to be $\mathscr{F}$ bordant if there is an $(n+1)$-dimensional $T$-manifold $N$ of type $(\mathscr{F}, \mathscr{F})$ such that the 
disjoint union of $M_{1}$ and $-M_{2}$ is the $T$-boundary of $N$. This equivalence relation on the set of $T$-manifolds of type $\mathscr{F}$ leads to a bordism theory denoted by $\mathfrak{U}_{*}^{T}[\mathscr{F}]$. We are mainly interested in the family "All" consisting of all $T$-slice types, of course $\mathfrak{U}_{*}^{T}$ is equal to $\mathfrak{H}_{*}^{T}[\mathrm{All}]$.

Let $\rho=[H ; U]$ be a $T$-slice type. A $T$-(complex)-vector bundle $E$ over a manifold $N$ is said to be of type $\rho$ if the set of points in $E$ having slice type $\rho$ is precisely $N$. We say that $E$ is a boundary if there is a $T$-vector bundle $F$ over $M$ such that $F$ is of type $\rho$ with $\partial M=N$ and $F \mid \partial M=E$. Bordism of bundles of type $\rho$ leads to the bundle bordism group $\mathfrak{U}_{*}^{T}[\rho]$. In fact $\mathfrak{A}_{*}^{T}[\rho]$ is isomorphic to $\mathfrak{A}_{*-k}(B \Gamma \rho)$ for some classifying space $B \Gamma \rho$ and some integer $k$, this was originally proved by Conner and Floyd, see $[3 ; 1.5]$ for a proof in the context of slice types.

If $\mathscr{F}^{\prime} \subseteq \mathscr{F}$ are families of $T$-slice types with $\mathscr{F}=\mathscr{F}^{\prime} \cup\{\rho\}$ then there is a natural $\mathfrak{A}_{*}$ module homomorphism

$$
v_{\rho}: \mathscr{U}_{*}^{T}[\mathscr{F}] \rightarrow \mathfrak{U}_{*}^{T}[\rho]
$$

given by assigning to $M$ the normal bundle of the points in $M$ with slice type $\rho$. This fits into a long exact sequence:

$$
\cdots \rightarrow \mathfrak{U}_{n}^{T}\left[\mathscr{F}^{\prime}\right] \rightarrow \mathfrak{U}_{n}^{T}[\mathscr{F}] \stackrel{v}{\rightarrow} \mathfrak{U}_{n}^{T}[\rho] \stackrel{\hat{\prime}}{\rightarrow} \mathfrak{U}_{n-1}^{T}\left[\mathscr{F}^{\prime}\right] \rightarrow \cdots,
$$

where $\partial(E)=S(E)$, the sphere bundle of $E$. This sequence provides an inductive method of calculating $\mathfrak{U}_{n}^{T}[\mathscr{F}]$, for any family $\mathscr{F}$ of $T$-slice types.

\section{Circle slice types}

The non-trivial irreducible $T$-modules are

$$
\ldots V_{-2}, V_{-1}, V_{1}, V_{2}, \ldots
$$

where $V_{j}$ denotes the complex space $\mathbb{C}$ with $t \in T$ acting by multiplication by $t^{j}$. We denote the non-trivial $\mathbb{Z} / m$-modules by

$$
V_{-1}, V_{-2}, \ldots, V_{-m+1}
$$

where $V_{j}$ is $\mathbb{C}$ with $t \in \mathbb{Z} / m \subseteq T$ acting by multiplications by $t^{j}$. The $T$-slice types take the form

$$
\begin{gathered}
{\left[T ; V_{k(1)} V_{k(2)} \ldots V_{k(n)}\right] \quad k(1) \geqq k(2) \geqq \ldots \geqq k(n) ; \quad n \geqq 0} \\
{\left[\mathbb{Z} / m ; V_{k(1)} V_{k(2)} \ldots V_{k(n)}\right] \quad 0>k(1) \geqq k(2) \geqq \ldots \geqq k(n)>m ; \quad n \geqq 0}
\end{gathered}
$$

It is sometimes convenient to write the latter in the form

$$
\left[\mathbb{Z} / m ; V_{-1}^{i(-1)} V_{-2}^{i(-2)} \ldots V_{-m+1}^{i(-m+1)}\right] \quad i(j) \geqq 0 .
$$


If we denote this $T$-slice type by $\rho$ then

$$
\mathfrak{U}_{*}^{T}[\rho] \cong \mathfrak{U}_{*-1-2 \sum i(-k)}\left(B(T /(\mathbb{Z} / m)) \times \prod_{k=1}^{m-1} B U_{i(-k)}\right) .
$$

Geometrically, an element $E \in \mathfrak{U}_{*}^{T}[\rho]$ is a $T$-vector bundle over some $T$-manifold $X$ for which $T_{x}=\mathbb{Z} / m$ for all $x \in X$. Furthermore, $E$ decomposes as a $T$-vector bundle, into

$$
E=E_{-1} \oplus E_{-2} \oplus \cdots \oplus E_{-m+1}
$$

where $\operatorname{dim}_{c} E_{k}=i(k)$ and $t \in \mathbb{Z} / m \subseteq T$ acts on each fibre of $E_{k}$ by multiplication by $t^{k}$.

Using this description of $E$ we can now describe the isomorphism ( $\dagger$ ) above. Since each point of $X$ has isotropy subgroup $\mathbb{Z} / m$ we obtain a $T$-fibration $X \rightarrow X / S$, where $S$ is $T /(\mathbb{Z} / m)$, and we denote the associated complex line bundle by $\vec{E}_{-m}$. Let $\bar{E}_{k}$ be $\left(E_{k} \otimes V_{-k}\right) / S$ and define $\bar{E}$ by

$$
\bar{E}=\bar{E}_{-1} \oplus \bar{E}_{-2} \oplus \cdots \oplus \bar{E}_{-m+1} \oplus \bar{E}_{-m}
$$

Then the isomorphism ( $\dagger$ ) is given by $E \rightarrow \bar{E}$.

To recover $E$ from $\bar{E}$ we may proceed as follows: Give $\bar{E}$ a T-action simply by letting $t \in T$ act on each fibre of $\bar{E}_{k}$ by multiplication by $t^{k}$. Now, the fixed point set of $S(\bar{E})$ under the $\mathbb{Z} / m \subseteq T$ action is precisely $X$ and the equivariant normal bundle of $X$ in $S(\bar{E})$ is $\mathrm{E}$. In other words the inverse of the isomorphism ( $\dagger$ ) is given by

$$
\bar{E} \rightarrow v S(\bar{E}) .
$$

\section{Finite isotropy}

Consider the family $\mathscr{F}$ consisting of all $T$-slice types with isotropy subgroup not equal to $T$, i.e.

$$
\mathscr{F}=\left\{[\mathbb{Z} / m ; U] ; m \geqq 1, U \text { is a } \mathbb{Z} / m \text { module with } U^{\mathrm{Z} / m}=\{0\}\right\}
$$

\section{Theorem}

$$
\mathfrak{A}_{*}^{T}[\mathscr{F}] \cong \bigoplus_{\rho \in F^{F}} \mathfrak{H}_{*+1}^{T}[\rho]
$$

The proof of this result is by induction over the elements of $\mathscr{F}$ and to achieve this we need to order the elements of $\mathscr{F}$. Suppose $\rho \in \mathscr{F}$ where

$$
\rho=\left[\mathbb{Z} / m ; V_{k(1)} V_{k(2)} \ldots V_{k(n)}\right]
$$

with $0>k(1) \geqq k(2) \geqq \cdots \geqq k(n)>-m$. We set

$$
\delta(\rho)=m, \quad d(\rho)=n+1, \quad(\delta+d)(\rho)=\delta(\rho)+d(\rho)
$$


that is, $\delta(\rho)$ is the order of the isotropy subgroup defining $\rho$ and $d(\rho)$ is the complex dimension of the slice type plus one. Now if $\sigma \in \mathscr{F}$ is another member with

$$
\sigma=\left[\mathbb{Z} / p ; V_{l(1)} V_{l(2)} \ldots V_{l(q)}\right]
$$

where $0>l(1) \geqq l(2) \geqq \cdots \geqq l(q)>-\rho$ then we say that $\rho<\sigma$ if either (i) $(\delta+d)(\rho)<(\delta+d)(\sigma)$ or (ii) $(\delta+d)(\rho)=(\delta+d)(\sigma)$ and $d(\rho)<d(\sigma)$ or (iii) $\delta(\rho)=\delta(\sigma), d(\rho)=d(\sigma)$ and $k_{1}<l_{1}$, or (iv) $\delta(\rho)=\delta(\sigma), d(\rho)=d(\sigma), k_{1}=l_{1}$ and $k_{2}<l_{2}$ etc.

This process totally orders the elements of $\mathscr{F}$ which we now denote by $\rho_{1}, \rho_{2}, \rho_{3}, \ldots$, with $\rho_{i}<\rho_{j}$ if $i<j$.

Lemma. $\quad \mathscr{F}_{j}=\left\{\rho_{i} ; i \leqq j\right\}$ is a family of $T$-slice types for all $j \geqq 1$.

The proof is easy and left for the reader.

To prove the Theorem we look at the exact sequence $\left(^{*}\right)$ for $\mathscr{F}_{j-1} \subseteq \mathscr{F}_{j}$, with $\mathscr{F}_{j}-\mathscr{F}_{j-1}=\left\{\rho_{j}\right\}$ :

$$
\cdots \rightarrow \mathfrak{U}_{n}^{T}\left[\mathscr{F}_{j-1}\right] \stackrel{i}{\rightarrow} \mathfrak{U}_{n}^{T}\left[\mathscr{F}_{j}\right] \stackrel{v_{j}}{\rightarrow} \mathfrak{U}_{n}^{T}\left[\rho_{j}\right] \stackrel{\hat{\partial}_{j}}{\rightarrow} \mathfrak{U}_{n-1}^{T}\left[\mathscr{F}_{j-1}\right] \rightarrow \cdots
$$

If $E \in \mathfrak{A}_{n}^{T}\left[\rho_{j}\right]$ we let $\bar{E}$ be the vector bundle determined by the isomorphism ( $\dagger$ ) of Section 3 with the action of $T$ as described in that section. It is an easy exercise to check that $S(\bar{E})$ is a $T$-manifold of type $\mathscr{F}_{j}$ so that we get a homomorphism

$$
\begin{aligned}
q_{j}: \mathfrak{U}_{n}^{T}\left[\rho_{j}\right] & \rightarrow \mathfrak{U}_{n}^{T}\left[\mathscr{F}_{j}\right] \\
\mathrm{E} & \rightarrow \mathrm{S}(\overline{\mathrm{E}})
\end{aligned}
$$

with $v_{j} q_{j}=1$. Thus $q_{j}$ splits the exact sequence and the required result follows by simple induction.

\section{Fixed point free actions}

For each element $\rho \in \mathscr{F}$ we define a $T$-slice type $e(\rho)$ as follows:

$$
e\left[\mathbb{Z} / m ; V_{k(1)} V_{k(2)} \ldots V_{k(n)}\right]=\left[T ; V_{k(1)} V_{k(2)} \ldots V_{k(n)} V_{-m}\right]
$$

where $0>k_{1} \geqq k_{2} \geqq \cdots \geqq k_{n}>-m$. We denote the set $\{e(\rho) ; \rho \in \mathscr{F}\}$ by $e \mathscr{F}$ and let $\mathscr{S} \mathscr{F}$ $=\mathscr{F} \cup e \mathscr{F}$. Note that $\mathscr{S} \mathscr{F}$ is a family of $T$-slice types.

$$
\text { Theorem. } \quad \mathfrak{U}_{*}^{T}[\mathscr{S} \mathscr{F}]=0 .
$$

To prove this let $\mathscr{S} \mathscr{F}_{2 n}=\left\{\rho_{j}, e\left(\rho_{j}\right) ; 1 \leqq j \leqq n\right\}$ and let $\mathscr{S} \mathscr{F}_{2 n+1}=\mathscr{S} \mathscr{F}_{2 n} \cup\left\{\rho_{n+1}\right\}$. It is easy to check that $\mathscr{S} \mathscr{F}_{j}$ is a family of $T$-slice types. Since $\mathfrak{U}_{*}^{T}\left[\rho_{j}\right] \cong \mathfrak{U}_{*}^{T}[e(\rho)]$ it follows by induction that

$$
\mathfrak{U}_{*}^{T}\left[\mathscr{S} \mathscr{F}_{2 n}\right]=0, \quad \mathfrak{U}_{*}^{T}\left[\mathscr{S} \mathscr{F}_{2 n+1}\right] \cong \mathfrak{U}_{*}^{T}\left[\rho_{n+1}\right]
$$


Corollary (E. Ossa [4]). If $M$ is a fixed point free T-manifold then $M$ is a T-boundary.

\section{Main result}

The next result, the main result, is now not surprising.

Theorem.

$$
\mathfrak{U}_{*}^{T} \cong \bigoplus_{\rho \in \mathscr{S} \mathscr{T}-\mathscr{S F}} \mathfrak{U}_{*}[\rho]
$$

where $\mathscr{S} \mathscr{T}$ is the set of T-slice types.

Note that

$$
\mathscr{S} \mathscr{T}-\mathscr{S} \mathscr{F}=\left\{[T: V] ; V \neq V_{k(1)} V_{k(2)} \ldots V_{k(n)} V_{-m} \text { with } 0>k_{1} \geqq k_{1} \geqq \cdots \geqq k_{n}>-m\right\}
$$

We need to order the elements of $\mathscr{S} \mathscr{T}$. If $\rho \in \mathscr{F}$ then we have defined $\delta(\rho)$ and $d(\rho)$, we now define

$$
\begin{aligned}
& \delta\left[T ; V_{k(1)} V_{k(2)} \ldots V_{k(n)}\right]=\max \{|k(i)| ; i=1,2, \ldots, n\} \\
& d\left[T ; V_{k(1)} V_{k(2)} \ldots V_{k(n)}\right]=n
\end{aligned}
$$

and note that $\delta e(\rho)=\delta(\rho) ; d e(\rho)=d(\rho)$. We may now order the elements of $\mathscr{S} \mathscr{T}-\mathscr{F}$ in the same manner as we ordered $\mathscr{F}$, i.e. by first using $\delta+d$, then $\delta$, then lexicographically. To obtain a total order on $\mathscr{S} \mathscr{T}$ we use the ordering of $\mathscr{S} \mathscr{T}-\mathscr{F}$ and $\mathscr{F}$ together with two additional conditions: if $\rho \in \mathscr{F}$ then we say that $\rho<e(\rho)$; if $\rho \in \mathscr{F}$, $\rho^{\prime} \in \mathscr{P} \mathscr{T}-\mathscr{F}$ with $e(\rho)>\rho^{\prime}$ then we say that $\rho>\rho^{\prime}$. Observe that if $\rho, \sigma \in \mathscr{F}$ then $\rho<\sigma$ if and only if $e(\rho)<e(\sigma)$.

Denote the elements of $\mathscr{S} \mathscr{T}$ by $\sigma_{1}, \sigma_{2}, \sigma_{3}, \ldots$, so that $\sigma_{i}<\sigma_{j}$ if $i<j$. It is easy to check that the set $\mathscr{S} \mathscr{T}_{j}=\left\{\sigma_{i} ; i \leqq j\right\}$ is a family of $T$-slice types.

The theorem is proved by induction, using the inductive hypothesis

$$
\mathfrak{A}_{*}^{T}\left[\mathscr{S}_{k}\right] \cong \bigoplus_{\sigma \in \mathrm{A}_{k}} \mathfrak{A}_{*}[\sigma]
$$

where

$$
A_{k}=\left\{\sigma \in \mathscr{S} \mathscr{T}_{k} ; \sigma \neq e(\rho) \text { for } \rho \in \mathscr{S} \mathscr{T}_{k} \cap \mathscr{F} \text { or } e(\sigma) \notin \mathscr{P} \mathscr{T}_{k}\right\}
$$

Look at the exact sequence (*) for $\mathscr{S} \mathscr{T}_{j-1} \subseteq \mathscr{S} \mathscr{T}_{j}, \mathscr{S} \mathscr{T}_{j}-\mathscr{S} \mathscr{T}_{j-1}=\left\{\sigma_{j}\right\}$ :

$$
\cdots \rightarrow \mathfrak{U}_{n}^{T}\left[\mathscr{S T}_{j-1}\right] \stackrel{i}{\rightarrow} \mathfrak{U}_{n}^{T}\left[\mathscr{S} \mathscr{T}_{j}\right] \stackrel{v_{j}}{\rightarrow} \mathfrak{U}_{n}^{T}\left[\sigma_{j}\right] \stackrel{\mathfrak{J}_{1}}{\rightarrow} \mathfrak{U}_{n-1}^{T}\left[\mathscr{P S}_{j-1}\right] \rightarrow \cdots
$$

Three cases arise:

(i) $\sigma_{j} \in e \mathscr{F}$. In this case $v_{j}=0$ and $\partial_{j}$ is injective. 
(ii) $\sigma_{j} \in \mathscr{F}$. In this case we define, as in Section $4, q_{j}(E)$ to be $S(\bar{E})$ for $E \in \mathfrak{A}_{*}^{T}\left[\sigma_{j}\right]$.

(iii) $\sigma_{j} \notin \mathscr{P} \mathscr{T}$. In this case if $E \in \mathfrak{X}_{n}^{T}\left[\sigma_{j}\right]$ then $S(E)$ is a fixed point free $T$-manifold. Consequently by the Corollary in Section 5 we have $S(E)=\partial(W)$ for some $T$ manifold $W$ of type $\mathscr{P} \mathscr{F} \cap \mathscr{P}_{j}$. Define $q_{j}(E)$ to be $D(E) \cup-W$ glued along their common boundary $S(E)$. This is a $T$-manifold of type $\mathscr{S T} \mathscr{T}_{j}$ and $v_{j} q_{j}(E)=E$.

This completes the proof of the Theorem.

\section{Geometric generators}

There is a more explicit, geometric, description of the splitting $q_{j}$ of case (iii) in Section 6 above, not using the results of Section 5. This provides explicit geometric generators of $\mathfrak{U}_{*}^{T}$ as alluded to in the introduction.

Throughout this section we assume that $\sigma$ is a $T$-slice type, not belonging to $\mathscr{P} \mathscr{F}$. Thus $\sigma$ may be written in the form

$$
\sigma=\left[T ; V_{k(1)}^{i(1)} V_{k(2)}^{i(2)} \ldots V_{k(p)}^{i(p)} V_{-l(1)}^{j(1)} V_{-l(2)}^{j(2)} \ldots V_{-l(q)}^{j(q)}\right]
$$

where

$$
k(1)>k(2)>\cdots>k(p)>0>-l(1)>-l(2)>\cdots>-l(q)
$$

and each of $i(1), i(2), \ldots, i(p), j(1), j(2), \ldots, j(q)$ are positive integers.

An element $E$ of $\mathfrak{U}_{*}^{T}[\sigma]$ may be written as

$$
E=E^{+} \oplus E^{-}=E_{1}^{+} \oplus E_{2}^{+} \oplus \cdots \oplus E_{p}^{+} \oplus E_{1}^{-} \oplus E_{2}^{-} \oplus \cdots \oplus E_{q}^{-}
$$

where $\operatorname{dim}_{C} E_{r}^{+}=i(r), \operatorname{dim}_{\mathrm{C}} E_{r}^{-}=j(r)$ and $t \in T$ acts on each fibre of $E_{r}^{+}, E_{r}^{-}$by multiplication by $t^{k(r)}, t^{-l(r)}$ respectively. The action of $T$ on the base space of $E$ is, of course, trivial.

Given $E \in \mathfrak{U}_{*}^{T}[\sigma]$ we define some $T$-manifolds in terms of $E$. First we define $\mathbb{C} T P\left(\mathbb{C}^{r} \oplus E\right)$, for any $r \geqq 0$, as follows:

$$
\mathbb{C} T P\left(\mathbb{C}^{r} \oplus E\right)=S\left(\mathbb{C}^{r} \oplus E^{+} \oplus E^{-}\right) /(T, \psi)
$$

where $(T, \psi)$ is an action of $T$ on $S\left(\mathbb{C}^{r} \oplus E^{+} \oplus E^{-}\right)$induced from an action of $T$ on $\mathbb{C}^{r} \oplus E^{+} \oplus E^{-}$for which $t \in T$ acts by multiplication by $t$ in each fibre of $\mathbb{C}^{r} \oplus E^{+}$and by multiplication by $t^{-1}$ in each fibre of $E^{-}$. Since the action $(T, \psi)$ is a free-unitary action on the unitary manifold $S\left(\mathbb{C}^{r} \oplus E^{+} \oplus E^{-}\right)$the quotient space is a unitary manifold in a natural way. We give $\mathbb{C} T P\left(\mathbb{C}^{r} \oplus E\right)$ a $T$-action which is the $T$-action induced from the original action of $T$ on $E=E^{+} \oplus E^{-}$and the trivial action on $\mathbb{C}^{r}$.

The above construction appears in [1] and is called a twisted projective space construction. We have used the symbols $\mathbb{C} T P$ to stand for complex twisted projective space.

Unfortunately this construction is not sufficient to provide us with all the generators of $\mathfrak{U}_{*}^{T}$, we need a second construction. 
For our second construction we need some more notation. Suppose that $E^{\prime}, E^{\prime \prime}$ are vector bundles over the same space, where $E^{\prime}$ is of real dimension $k$ and $E^{\prime \prime}$ is of real dimensions $l$. Then, by $S\left(E^{\prime}\right) \oplus S\left(E^{\prime \prime}\right)$ we mean the $S^{k-1} \times S^{l-1}$ bundle whose fibre over a point $x$ is $S\left(E_{x}^{\prime}\right) \times S\left(E_{x}^{\prime \prime}\right)$.

For $E \in \mathfrak{A}_{*}^{T}[\sigma]$ we define $\widetilde{\mathbb{C T P}}(\mathbb{C} \oplus E)$ as follows:

$$
\widetilde{\mathbb{C} T P}(\mathbb{C} \oplus E)=\left(S\left(\mathbb{C} \oplus \hat{E}^{-}\right) \oplus S\left(\mathbb{C} \oplus E^{+} \oplus E_{q}^{-}\right)\right) /(T \times T, \psi)
$$

where $\hat{E}^{-}=E_{1}^{-} \oplus E_{2}^{-} \oplus \cdots \oplus E_{q-1}^{-}$and $(T \times T, \psi)$ is the action determined as follows:

$$
\psi\left((t, s),\left(e_{0}, e_{-}\right)_{x}\left(f_{0}, f_{+}, f_{-}\right)_{x}\right)=\left(t^{-1} e_{0}, t^{-1} e_{-}\right)_{x}\left(s f_{0}, s t f_{+}, s^{-1} t^{-1} f_{-}\right)_{x}
$$

for

$$
(t, s) \in T \times T,\left(e_{0}, e_{-}\right)_{x}\left(f_{0}, f_{+}, f_{-}\right)_{x} \in S\left(\left(\mathbb{C} \oplus \hat{E}^{-}\right)_{x}\right) \times S\left(\left(\mathbb{C} \oplus E^{+} \oplus E_{q}^{-}\right)_{x}\right)
$$

We give $\overparen{\mathbb{C} T P}(\mathbb{C} \oplus E \oplus F)$ the induced unitary structure and a $T$-action induced by the original action of $T$ on $E=E^{+} \oplus \hat{E}^{-} \oplus E_{q}^{-}$and the trivial action on $\mathbb{C}$.

Remarks. (1) $\overparen{\mathbb{C} T P}(\mathbb{C} \oplus E)$ is a $\mathbb{C} T P\left(\mathbb{C} V_{-l(1)}^{j(1)} V_{-l(2)}^{j(2)} \ldots V_{-l(q-1)}^{j(q-1)}\right)$ fibre bundle over $\mathbb{C} T P\left(\mathbb{C} \oplus E^{+} \oplus E_{q}^{-}\right)$.

(2) $\mathbb{C} T P\left(\mathbb{C}^{r} \oplus E\right)$ is a $\mathbb{C} T P\left(\mathbb{C}^{r} V_{k(1)}^{i(1)} V_{k(2)}^{i(2)} \cdots V_{k(p)}^{i(p)} V_{-l(1)}^{j(1)} V_{-l(2)}^{j(2)} \cdots V_{-l(q)}^{j(q)}\right)$ fibre bundle over the base space manifold of $E$.

To obtain a geometric description of the splitting $q_{j}$ we proceed as follows. If $E \in \mathfrak{A}_{*}^{T}\left[\sigma_{j}\right]$ where $\sigma_{j} \notin \mathscr{S} \mathscr{F}$ then we define

$$
\begin{aligned}
q_{j}(E) & =\widetilde{\mathbb{C} T P}(\mathbb{C} \oplus E) \text { if } j(q)=1 \text { and } l(q)>k(1) \\
& =\mathbb{C} T P(\mathbb{C} \oplus E) \text { otherwise }
\end{aligned}
$$

It is not difficult to check that $q_{j}(E)$ is a $T$-manifold of type $\mathscr{S} \mathscr{T}_{j}$, and that $v_{j} q_{j}(E)=E$. Thus by the main result of Section 6 we deduce that these manifolds provide geometric generators of $\mathfrak{A}_{*}^{T}$.

An explicit list of generators may be easily written down using the fact that the elements

$$
\eta_{n} \otimes V_{k}, \quad n \geqq 0, \quad k \in \mathbb{Z}-\{0\}
$$

multiplicatively generate $\oplus_{\rho \in \mathscr{G} \mathscr{T}} \mathfrak{A}_{*}^{T}[\rho]$, where $\eta_{n}$ denotes the standard Hopf complex line bundle over $\mathbb{C} P^{n}$. We leave this for the reader to do.

\section{An application}

Using the geometric description of generators of $\mathfrak{A}_{*}^{T}$ we may easily prove a result 
about the $T_{y}$ genus of a unitary $T$-manifold. This was first proved by $\mathrm{C}$. Kosniowski in [2], A. Hattori and $H$. Taniguchi gave a proof in [1], subsequently there have been many other proofs and variations of the result.

Recall that the $T_{y}$ genus is the genus associated to the formal power series in $t$ :

$$
t(\exp (t(1+y))+y)(\exp (t(1+y))-1)^{-1}
$$

Theorem. Let $M$ be a T-manifold, then

$$
\begin{aligned}
T_{y}(M) & \sum_{F \in \text { Fix }}(-y)^{d(+, F)} T_{y}(F) \\
& =\sum_{F \in \text { Fix }}(-y)^{d(-, F)} T_{y}(F) .
\end{aligned}
$$

The meaning of the symbols $d( \pm, F)$ is as follows. For a component $F$ of Fix the fixed point set, the normal bundle $v(F, M)$ decomposes as

$$
v(F, M)=\bigoplus_{j \in \mathbf{Z}-\{0\}} v_{j}(F)
$$

where $t \in T$ acts on each fibre of $v_{j}(F)$ by multiplication by $t^{j}$. We define

$$
\begin{aligned}
& d(+, F)=\operatorname{dim}_{\mathrm{C}}\left(\bigoplus_{j>0} v_{j}(F)\right) \\
& d(-, F)=\operatorname{dim}_{\mathbf{C}}\left(\bigoplus_{j<0} v_{j}(F)\right) .
\end{aligned}
$$

In order to prove the Theorem it is sufficient to check that the formulae hold for $\widetilde{\mathbb{C} T P}(\mathbb{C} \oplus E)$ and $\mathbb{C} T P(\mathbb{C} \oplus E)$ for $E \in \bigoplus_{\rho \in \mathscr{G}} \mathfrak{I}_{*}^{T}[\rho]$ since these generate $\mathfrak{A}_{*}^{T}$.

Now, the $T_{y}$ genus is multiplicative for fibre bundles as are the expressions on the right in the Theorem. Hence, by the two remarks made in the last section it is sufficient to check the formulae for $\mathbb{C} T P\left(\mathbb{C} V_{+} V_{-}\right)$where

$$
V_{+}=V_{k(1)}^{i(1)} V_{k(2)}^{i(2)} \ldots V_{k(p)}^{i(p)}, \quad V_{-}=V_{-l(1)}^{j(1)} V_{\rightarrow l(2)}^{j(2)} \ldots V_{-l(q)}^{j(q)}
$$

for some choice of indices. It is in fact more convenient to check the formulae for $\widetilde{\mathbb{C T P}}\left(\mathbb{C}^{r} V_{+} V_{-}\right)$. In the case of a semifree $T$-action, i.e. the case in which $V_{+}=V_{1}^{a}$ and $V_{-}$ $=V_{-1}^{b}$, it is not difficult to check the formulae using the fact that

$$
T_{y}\left(\mathbb{C} T P\left(\mathbb{C}^{r} V_{1}^{a} V_{-1}^{b}\right)\right)=\left((-y)^{b}-(-y)^{a+r}\right) /(1-(-y))
$$

This latter fact is just a direct calculation of the $T_{y}$ genus of a manifold, details are given in Lemma 3.1 of $[1]$ where $\mathbb{C} T P\left(\mathbb{C}^{r} V_{1}^{a} V_{-1}^{b}\right)$ is denoted by $\mathbb{C} P_{a+r, b}$.

The general case is done by induction on the dimension of $\mathbb{C}^{r} V_{+} V_{-}$. To do this we 
put another $T$-action, say $(T, \psi)$, on $\mathbb{C} T P\left(\mathbb{C} V_{+} V_{-}\right)$so that this new action is semifree:

$$
\psi\left(t,\left(z_{0}, z_{+}, z_{-}\right)\right)=\left(z_{0}, t^{+1} z_{x}, t^{-1} z_{-}\right)
$$

for $t \in T,\left(z_{0}, z_{1}, z_{1}\right) \in \mathbb{C} V_{+} V_{-}$. Using this semifree action we reduce the problem to checking that the formulae hold for $\mathbb{C T P}\left(V_{+} V_{-}\right)$, induction completes this. Details are left for the reader.

\section{REFERENCES}

1. A. Hattori and H. TANiguchi, Smooth $S^{1}$-action and bordism, J. Math. Soc. Japan 24 (1972), 701-731.

2. C. Kosniowski, Applications of the holomorphic Lefschetz formula, Bull. Lond. Math. Soc. 2 (1970), 43-48.

3. C. Kosniowski, Actions of finite abelian groups (Pitman, London-San Francisco-Melbourne, 1978).

4. E. Ossa, Fixpunktfreie $S^{1}$-Aktionen, Math. Ann. 186 (1970), 45-52.

School of Mathematics

THE UNIVERSITY

NeWCASTlE UPON TyNE

ENGLAND
School of Mathematics UNIVERSITY OF KHARTOUM

Khartoum 